\title{
INTEGRAL broadband X-ray spectrum of the intermediate polar V709 Cassiopeiae
}

\author{
M. Falanga ${ }^{1,2}$, J. M. Bonnet-Bidaud ${ }^{1}$, and V. Suleimanov ${ }^{3,4,5}$ \\ ${ }^{1}$ CEA Saclay, DSM/DAPNIA/Service d'Astrophysique (CNRS FRE 2591), 91191 Gif-sur-Yvette, France \\ e-mail: mfalanga@cea.fr \\ 2 Unité mixte de recherche Astroparticule et Cosmologie, 11 place Berthelot, 75005 Paris, France \\ 3 Kazan State University, Kremlevskaya str. 18, 420008 Kazan, Russia \\ 4 Astronomy Division, PO Box 3000, 90014 University of Oulu, Finland \\ 5 Kazan Branch of Isaac Newton Institute, Santiago, Chile
}

Received 6 August 2005 / Accepted 14 September 2005

ABSTRACT

We present the hard X-ray time-averaged spectrum of the intermediate polar V709 Cas observed with INTEGRAL. We performed the observation using data from the IBIS/ISGRI instrument in the 20-100 keV energy band and from JEM-X at lower energy (5-20 keV). Using different multi-temperature and density X-ray post-shock models we measured an improved post-shock temperature of $\sim 40 \mathrm{keV}$ and estimated the V709 Cas mass to be $0.82_{-0.25}^{+0.12} M_{\odot}$. We compare the resulting spectral parameters with previously reported BeppoSAX and RXTE observations. Key words. binaries: general - stars: individual: V709 Cas - X-rays: stars

\section{Introduction}

V709 Cas (RX J0028+5917) is a member of the intermediate polars (IP) systems, a sub-class of magnetic cataclysmic variables. This system consists of an accreting white dwarf (WD) and a low-mass late type main sequence companion star. The accretion onto V709 Cas is believed to be driven through Roche-lobe overflow, where the accretion flow from the secondary proceeds towards the IP through an accretion disk, until it reaches the magnetospheric radius. Here the material attaches to the magnetic field lines and follows them almost radially at free-fall velocity towards the magnetic poles of the IP surface. At some distance from this surface, the accretion flow undergoes a strong shock, below which material settles onto the IP, releasing X-rays as it cools by thermal bremsstrahlung processes (see reviews Aizu 1973; Cropper 1990; Patterson 1994). According to this standard model the spectra of the X-ray post-shock emitting region has a multitemperature and density structure (e.g., Cropper et al. 1999). In order to measure the maximal shock temperature, hight energy observatories like INTEGRAL are needed. In most cases the temperature of the post-shock region of the accretion column of IPs are in the order of $\sim 10-60 \mathrm{keV}$. A hard X-ray study can also be used to estimate the IP mass by measuring the maximum temperature of the post-shock plasma (Suleimanov et al. 2005). Using the INTEGRAL/RXTE data on the IP V1223 Sgr Revnivtsev et al. (2004) studied such a broadband X-ray spectrum to determine the shock parameters and the IP mass.

The X-ray source V709 Cas was discovered and identified as an IP from the ROSAT All sky Survey (Haberl \& Motch 1995) and has been extensively studied with ROSAT
(Haberl \& Motch 1995; Motch et al. 1996; Norton et al. 1999), with a joint $R X T E / B e p p o S A X$ X-ray observation (De Martino et al. 2001) and using optical spectroscopy and photometry (Bonnet-Bidaud et al. 2001; Kozhevnikov 2001). The pulse period was found to be $312.8 \mathrm{~s}$ (Haberl \& Motch 1995; Norton et al. 1999) and a detailed spectroscopic study finally concluded on a $5.34 \mathrm{~h}$ orbital period after a previous ambiguity between $5.4 \mathrm{~h}$ and $4.5 \mathrm{~h}$ (Bonnet-Bidaud et al. 2001).

We present here the first INTEGRAL results of a broadband spectrum study, from 5-100 keV, where the energies above $20 \mathrm{keV}$ are based on hard X-ray IBIS/ISGRI observations of V709 Cas.

\section{Observations and data analysis}

The present dataset was obtained during the Target of Opportunity (ToO) A02 INTEGRAL (Winkler et al. 2003) observation of the Cas A region performed from 5-6 and 7-9 December 2004 (53 345.6-53346.8 and 53 347.8-53 349.8 MJD), i.e. from part of INTEGRAL satellite revolutions 262 and 263. We use data from the IBIS/ISGRI (20-100 keV) coded mask imager (Ubertini et al. 2003; Lebrun et al. 2003) for a total exposure of $181.9 \mathrm{ks}$ and from the JEM-X (5$20 \mathrm{keV}$ ) monitor (Lund et al. 2003) for a total exposure time of $82 \mathrm{ks}$. For ISGRI, the data were extracted for all pointings with a source position offset $\leq 7^{\circ}$, and for JEM-X with an offset $\leq 3.5^{\circ}$. The spectrometer (SPI) (Roques et al. 2003) was not used to extract the hard X-ray spectrum due to the lower sensitivity of this instrument with respect to IBIS/ISGRI for a weak source below $100 \mathrm{keV}$. Above $90 \mathrm{keV}$, V709 Cas was not consistently detected in a single exposure or in the total 


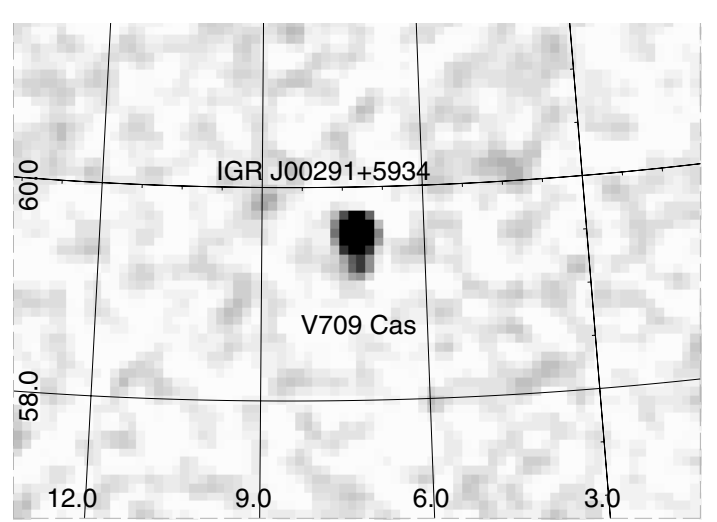

Fig. 1. The 20-40 keV IBIS/ISGRI mosaicked and deconvolved sky image of the $\sim 182 \mathrm{ks}$ observation. Image size is $\sim 12^{\circ} \times 5^{\circ}$, centered at V709 Cas position. The pixel size is $5^{\prime}$. V709 Cas and IGR J00291+5934 at a separation of $20^{\prime}$ were detected at a significance of $\sim 12.6 \sigma$ and at $\sim 82.8 \sigma$, respectively.

exposure. Data reduction was performed using the standard Offline Science Analysis (OSA) version 4.2 (Courvoisier et al. 2003). The algorithms used in the spatial and spectral analysis are described in Goldwurm et al. (2003).

\section{Results}

\subsection{ISGRI Imaging}

Figure 1 shows a significance map around the source V709 Cas in the $20-40 \mathrm{keV}$ energy range. Single pointings were deconvolved and analyzed separately, and then combined in mosaic images. Two sources are clearly detected at a significance level of $12.6 \sigma$ for V709 Cas and $82.8 \sigma$ for IGR J00291+5934, a newly discovered millisecond pulsar in outburst (Falanga et al. 2005). In the 40-80 keV energy band, the confidence level was $5 \sigma$ for V709 Cas and $46 \sigma$ for IGR J00291+5934. At energies above $100 \mathrm{keV}$ V709 Cas was not detected at a statistically significant level either in single exposures or in the total exposure time. To obtain precise source locations we simultaneously fitted the ISGRI point spread function to the two close sources. We obtained a position for V709 Cas at $\alpha_{\mathrm{J} 2000}=00^{\mathrm{h}} 28^{\mathrm{m}} 55^{\mathrm{s}} .29$ and $\delta_{\mathrm{J} 2000}=59^{\circ} 16^{\prime} 14^{\prime \prime}$. 0 . The position of IGR J00291+5934 is given by $\alpha_{\mathrm{J} 2000}=00^{\mathrm{h}} 29^{\mathrm{m}} 02^{\mathrm{s}} .92$ and $\delta_{\mathrm{J} 2000}=59^{\circ} 34^{\prime} 06^{\prime \prime} 4$. The source position offsets with respect to the optical catalog positions (Downes et al. 1997; Fox \& Kulkarni 2004) are 1'.4 for V709 Cas and 0.2 for IGR J00291+5934. The errors are 1.5 and 0.2 for V709 Cas and IGR J00291+5934. These are within the $90 \%$ confidence level assuming the source location error given by Gros et al. (2003). The derived angular distance between the two sources is $\sim 20^{\prime}$. Due to the fact that INTEGRAL is able to image the sky at high angular resolution $\left(12^{\prime}\right.$ for ISGRI and $3^{\prime}$ for JEM-X), we were able to clearly distinguish and isolate the high-energy fluxes from the two sources separately. This allows us to isolate and study the X-ray emission of V709 Cas during the outburst of the IGR J00291+5934 pulsar. The lack of contamination by the pulsar outburst was verified by building V709 Cas light curves in different energy ranges. While during the observation the pulsar showed a significant decay with an e-folding time of $\sim 6.6$ day (Falanga et al. 2005), the V709 Cas (20-80 keV) light curve remains stable with a constant mean count rate of $\sim 0.6 \pm 0.09$.
Table 1. Best fit parameters of the phase-averaged X-ray spectra.

\begin{tabular}{|c|c|c|}
\hline $\begin{array}{l}\text { Dataset } \\
\text { Energy range }\end{array}$ & $\begin{array}{l}\text { JEM-X/ISGRI } \\
(5-100) \mathrm{keV}\end{array}$ & $\begin{array}{l}\text { ISGRI } \\
(20-100) \mathrm{keV}\end{array}$ \\
\hline Model & \multicolumn{2}{|c|}{ BREMSSTRAHLUNG } \\
\hline $\begin{array}{l}k T_{\text {Brems }}(\mathrm{keV}) \\
\chi^{2} / \text { d.o.f. } \\
F_{\mathrm{X}}\left(\mathrm{erg} \mathrm{cm}^{-2} \mathrm{~s}^{-1}\right) \\
\end{array}$ & $\begin{array}{l}26.7_{+10.4}^{-6.7} \\
5.3 / 12 \\
1.2 \times 10^{-10}\end{array}$ & $\begin{array}{l}25.5_{-6.1}^{+9.3} \\
5.8 / 9 \\
0.52 \times 10^{-10}\end{array}$ \\
\hline Model & \multicolumn{2}{|c|}{ MEKAL } \\
\hline $\begin{array}{l}k T_{\text {Max }}(\mathrm{keV}) \\
\chi^{2} / \text { d.o.f. } \\
F_{\mathrm{X}}\left(\mathrm{erg} \mathrm{cm}^{-2} \mathrm{~s}^{-1}\right)\end{array}$ & $\begin{array}{l}25.7_{-6.4}^{+9.7} \\
5 / 12 \\
1.3 \times 10^{-10}\end{array}$ & $\begin{array}{l}24.6_{-5.8}^{+8.6} \\
5.8 / 9 \\
0.57 \times 10^{-10}\end{array}$ \\
\hline Model & \multicolumn{2}{|c|}{$\begin{array}{c}\text { POST-SHOCK } \\
\end{array}$} \\
\hline $\begin{array}{l}M_{\mathrm{wd}}\left(M_{\odot}\right) \\
k T_{\text {shock }}^{\dagger}(\mathrm{keV}) \\
\chi^{2} / \text { d.o.f. } \\
F_{\mathrm{x}}\left(\mathrm{erg} \mathrm{cm}^{-2} \mathrm{~s}^{-1}\right)\end{array}$ & $\begin{array}{l}0.82_{-0.25}^{+0.12} \\
39.38 \\
5 / 12 \\
1.2 \times 10^{-10} \\
\end{array}$ & $\begin{array}{l}0.85_{-0.15}^{+0.18} \\
41.3 \\
6.3 / 9 \\
0.95 \times 10^{-10} \\
\end{array}$ \\
\hline
\end{tabular}

\subsection{Spectral analysis}

The spectral analysis was done using XSPEC version 11.3 (Arnaud 1996), combining the 20-100 keV ISGRI data with the simultaneous 5-20 keV JEM-X data. Due to the short exposure time of JEM-X, and therefore lower statistics, we rebinned this data in a 5 channel energy response matrix. A constant factor was included in the fit to take into account the uncertainty in the cross-calibration of the instruments. A systematic error of $2 \%$ was applied to the JEM-X/ISGRI spectra which corresponds to the current uncertainty in the response matrix. All spectral uncertainties in the results are given at a $90 \%$ confidence level for single parameters.

The joint JEM-X/ISGRI (5-100 keV) broadband spectrum was first fitted with a simple optically thin thermal bremsstrahlung. The data fitted with $\chi^{2} /$ d.o.f. $=5 / 12$, and the plasma temperature was found around $27 \mathrm{keV}$. In order to compare with previously reported measurements (e.g., De Martino et al. 2001), we also fit the plasma emission model MEKAL. We found a consistent plasma temperature value with the previous thermal bremsstrahlung fit, where the flux in the $5-100 \mathrm{keV}$ energy range is $1.2 \times 10^{-10} \mathrm{erg} \mathrm{cm}^{-2} \mathrm{~s}^{-1}$ (see Table 1). During our INTEGRAL observation the source flux in the $3-100 \mathrm{keV}$ was $\sim 1.4$ times smaller than that observed in July 1998 by BeppoSAX and $\sim 2$ times smaller by RXTE (De Martino et al. 2001; Suleimanov et al. 2005). The obtained value of the temperature parameter $(26 \mathrm{keV})$ is significantly smaller than that obtained by De Martino et al. (2001) from BeppoSAX data (42 keV) and RXTE data (36 keV) using the same model. De Martino et al. (2001) found the same plasma temperature, $\sim 26 \mathrm{keV}$, only by including a reflection model in the fit. We attempted to test the hypothesis of a Compton reflection in the spectrum including the reflection model from Magdziarz \& Zdziarski (1995). The lack of statistics in the low energy part of the JEM-X data prevent us from determining significant reflection parameters. The best fit parameters using only the hight energy ISGRI data alone agree with the value from the joint JEM-X/ISGRI spectrum. This confirms the importance of the high energy observation to determine the post-shock temperature above $20 \mathrm{keV}$. 
However, from the standard accretion shock model, when the matter falls onto the WD, the X-ray post-shock emission region is expected to show a multi-temperature and multi-density structure. To fit the spectrum we therefore use a more physically motivated model where the X-ray emission is determined through a density and temperature gradient along the emission region. The broadband time-averaged spectrum is then given by summing local bremsstrahlung spectra in the region between the WD surface and the shock distance, $z_{0}$. We use the same geometrical model described by Suleimanov et al. (2005), where the observed flux is given by Zombeck (1990):

$F_{E}=9.52 \times 10^{-38}$

$\int_{R_{w d}}^{z_{0}}\left(\frac{\rho(z)}{\mu m_{\mathrm{H}}}\right)^{2} T(z)^{-1 / 2}\left(\frac{E}{k T(z)}\right)^{-0.4} \exp \left(-\frac{E}{k T(z)}\right) \mathrm{d} z$,

allowing the density profile, $\rho(z)$, and temperature profile, $T(z)$, to vary in the post-shock region (e.g., see Suleimanov et al. 2005; Cropper et al. 1999). In the flux equation, $\mu=0.62$ is the mean molecular weight of fully ionized accreting matter, $m_{\mathrm{H}}$ is the hydrogen mass and $k$ is the Boltzmann constant. The only two parameters in this model is now the WD mass, $M_{\mathrm{wd}}$, and the accretion rate by unit of surface $a$. From the best fit, using a standard local accretion rate of $a=1.0 \mathrm{~g} \mathrm{~cm}^{-2} \mathrm{~s}^{-1}$, we found $M_{\mathrm{wd}}=0.82 M_{\odot}$ corresponding to a shock temperature of $39 \mathrm{keV}$ (Table 1). The $\chi^{2}$ value is comparable with one of the simple bremsstrahlung model, however the post-shock model represents in more detail the physical processes in the WD emission region. Absorption by neutral hydrogen and a partial absorber have not been included in these models since they have no significant effect above $5 \mathrm{keV}$. Table 1 gives the best fit parameters of this column model, for the ISGRI and combined JEM-X/ISGRI data sets. In Fig. 2 we present the $v F_{v}$ spectrum of the entire observation, plotted together with the residuals in units of $\sigma$ with respect to the best fit post-shock model.

\subsubsection{Spectral analysis: models}

Different models for the structure of the accretion column have been put forward based on assumptions of constant pressure (Frank et al. 2002), influence of the magnetic field (Wu et al. 1994) or gravitational potential (Cropper et al. 1999). In an effort to also evaluate the influence of these different assumptions, a phenomenological fit with a variable emission measure can be performed. Each model is characterized by a powerlaw type density and temperature profiles along the accretion column. Assuming profiles with $\left(T / T_{\text {shock }}\right)=\left(x / x_{\text {shock }}\right)^{\alpha}$ and $\left(\rho / \rho_{\text {shock }}\right)=\left(x / x_{\text {shock }}\right)^{\beta}$, it can be shown that the emission measure $E M=\int_{R_{w d}}^{z_{0}} \rho(z)^{2} A \mathrm{~d} z$ is defined as:

$E M=\left(T / T_{\text {shock }}\right)^{\Gamma}$ with $\Gamma=\frac{(2 \beta+1)}{\alpha}$.

The $E M$ therefore also follows a power-law in temperature with an index $\Gamma$ and the column spectrum can be described with a power-law multi-temperature plasma emission model such as the XSPEC-CEMKL. The parameters $\alpha$ and $\beta$ have been determined by fitting the published profiles of the different accretion shock structure models and are reported in Table 2. To determine the post-shock temperature, the JEM-X/ISGRI spectrum

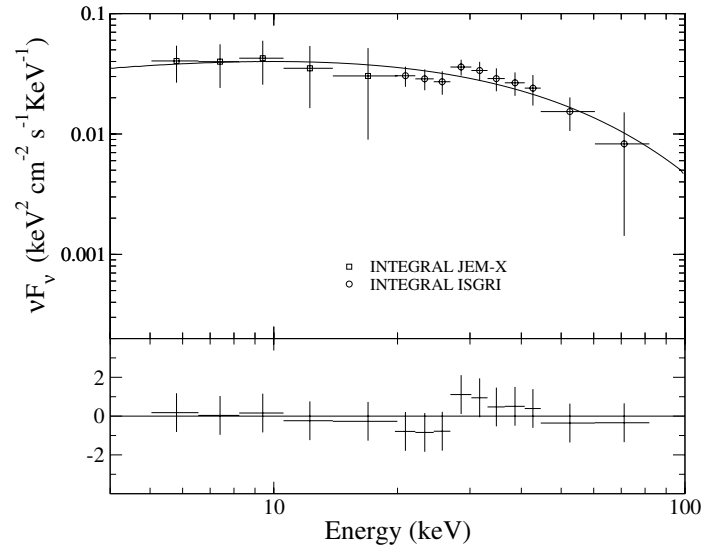

Fig. 2. The unfolded combined INTEGRAL JEM-X/ISGRI (5$100 \mathrm{keV}$ ) spectrum of V709 Cas with the best-fit post-shock model (line). Residuals between the data and model are shown in the bottom panel in units of sigma.

was fitted using CEMKL and fixing the model dependent powerlaw index, $\Gamma$, and the results are given in Table 2.

\section{Conclusions}

A first broadband spectrum result on V709 Cas was studied by De Martino et al. (2001) using BeppoSAX and RXTE data. We report here the first 5-100 keV high energy INTEGRAL spectrum result of V709 Cas. We found a Bremsstrahlung temperature around $26 \mathrm{keV}$ which is in agreement with a post-shock temperature around $40 \mathrm{keV}$ (see Fig. 3). The theoretical spectrum of the post-shock model shown in Fig. 3 is calculated using Eq. (1), where the bremsstrahlung spectrum is the best fit using the same equation as a bremsstrahlung model. The Bremsstrahlung temperature is lower than $T_{\text {shock }}$ since $T_{\text {Brems }}$ represent more a weighted mean value between the temperature distribution from the IP surface to the shock hight $z_{0}$. Using JEM-X data in the energy band 5-20 keV and statistically significant ISGRI data in the energy range from $20-100 \mathrm{keV}$ we estimated the source mass to be $0.82_{-0.25}^{+0.12} M_{\odot}$. Using the broad band RXTE spectrum Suleimanov et al. (2005) found within the error bars the same IP mass. The maximum shock temperature reported in Table 1 is calculated from:

$T_{\text {shock }}=\frac{3}{8} \frac{\mu G M_{\mathrm{wd}} m_{\mathrm{H}}}{k R_{\mathrm{wd}}}$.

Using different accretion shock structure models we found the same post-shock temperature (see Table 2). The IP radius was calculated from the Nauenberg (1972) WD mass-radius relation and we obtained a radius of $R_{\mathrm{wd}}=(0.68 \pm 0.13) \times 10^{9} \mathrm{~cm}$. The obtained estimation of the IP mass is slightly larger than that obtained by Bonnet-Bidaud et al. (2001). These authors inferred the mass estimation using optical spectroscopy, i.e. Balmer absorption lines, believed to be from the WD photosphere.

We estimate the accretion rate from the X-ray luminosities of the INTEGRAL observation. For the source distance we use the range 210-250 pc (Bonnet-Bidaud et al. 2001). The absorption corrected broad band X-ray flux of V709 Cas in the $0.5-100 \mathrm{keV}$ energy range is $F_{0.5-100 \mathrm{keV}}=1.6 \times$ $10^{-10} \mathrm{erg} \mathrm{cm}^{-2} \mathrm{~s}^{-1}$, therefore the isotropic luminosity of one 
Table 2. Best fit parameters from the JEM-X/ISGRI data in the 5-100 keV energy range.

\begin{tabular}{lllllllr}
\hline \hline Model & $\alpha$ & $\beta$ & $\Gamma$ & $B$-Field (MG) & $k T_{\text {shock }}(\mathrm{keV})$ & $\chi^{2} /$ d.o.f. & $F_{\mathrm{x}}\left(\mathrm{erg} \mathrm{cm}^{-2} \mathrm{~s}^{-1}\right)$ \\
\hline Frank et al. (2002) & 0.4 & -0.4 & 0.5 & - & $39.9_{+12.1}^{-12.3}$ & $4.91 / 12$ & $1.36 \times 10^{-10}$ \\
Wu et al. (1994) & 0.341 & -0.399 & 0.592 & - & $39.02_{+1.8}^{-11.8}$ & $4.8 / 12$ & $1.35 \times 10^{-10}$ \\
Wu et al. (1994) & 0.389 & -0.452 & 0.247 & 30 & $42.9_{+13.8}^{-13.8}$ & $5.07 / 12$ & $1.4 \times 10^{-10}$ \\
Suleimanov et al. (2005) & 0.312 & -0.433 & 0.430 & - & $40.6_{+13.1}^{-12.6}$ & $4.95 / 12$ & $1.37 \times 10^{-10}$ \\
\hline
\end{tabular}

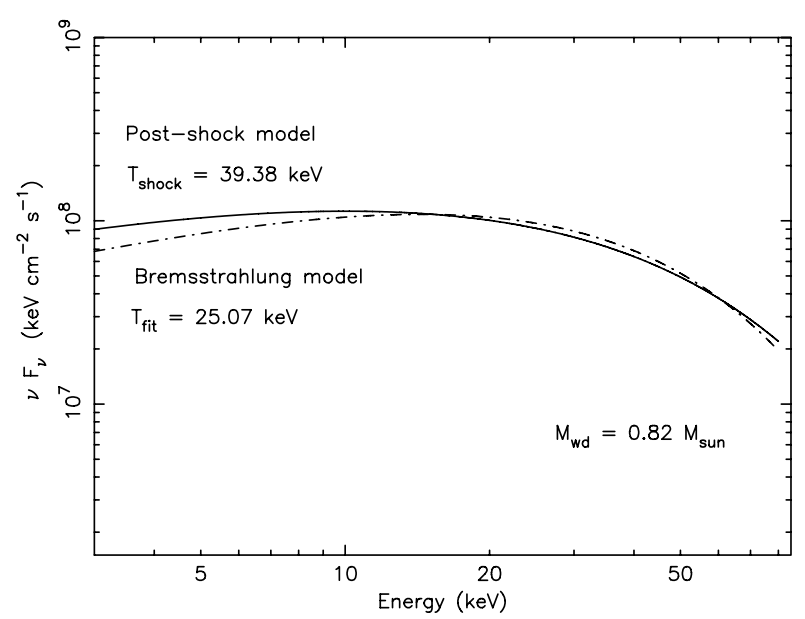

Fig. 3. The figure shows the agreement between the found value of the shock temperature from the post-shock model (see Sect. 3.2) with the best fit value using Eq. (1) as a Bremsstrahlung model.

accretion column is $L_{\mathrm{x}, 1}=4 \pi D^{2} F_{0.5-100 \mathrm{keV}}=0.84-1.2 \times$ $10^{33} \mathrm{erg} \mathrm{s}^{-1}$. From the Bremsstrahlung model the emission measure of one emitting accretion column is $E_{m, 1}=\int N_{\mathrm{e}}^{2} \mathrm{~d} V=$ $4.72 \times 10^{12} 4 \pi D^{2} \mathrm{~cm}^{-3}$, where $N_{\mathrm{e}}$ is the number density of the electron density in a hot emitting plasma. The volume emission measure therefore is $E_{m, 1} \approx 2.5-3.5 \times 10^{55} \mathrm{~cm}^{-3}$, consistent with the value found by De Martino et al. (2001). As the X-ray emission is the major channel of energy loss in the post-shock region we derived the mass accretion rate $\dot{M}$

$\dot{M} \approx \frac{3}{8} \frac{\mu m_{\mathrm{H}} G 2 L_{\mathrm{x}, 1}}{k T_{\text {shock }}}$.

The factor 2 is to account for two emitting magnetic poles of the IP. Substituting measured values we obtained $\dot{M}=0.82-1.2 \times$ $10^{16} \mathrm{~g} \mathrm{~s}^{-1}$. Using $m_{\mathrm{wd}}=0.82$ in units of the solar mass, $R_{\mathrm{wd}}=$ $0.68 \times 10^{9} \mathrm{~cm}$ and the $\dot{M}$ we evaluated the height of the standing shock $h_{\mathrm{S}}$ and the number density of electrons in the post-shock region $N_{\mathrm{e}}$ following Warner (1995).

$N_{\mathrm{e}}=3.1 \times 10^{15} \dot{M}_{16}\left(\frac{m_{\mathrm{wd}}}{M_{\odot}}\right)^{1 / 2} R_{9}^{-3 / 2} f_{-3}^{-1} \mathrm{~cm}^{-3}$

$h_{\mathrm{s}}=9.6 \times 10^{7} m_{\mathrm{wd}}\left(\frac{N_{\mathrm{e}}}{10^{16} \mathrm{~cm}^{-3}}\right)^{-1} R_{9}^{-1} \mathrm{~cm}$,

where $\dot{M}_{16}$ is the mass accretion rate of $10^{16} \mathrm{~g} \mathrm{~s}^{-1}, R_{9}$ is the IP radius in the units of $10^{9} \mathrm{~cm}$, and $f$ is the fraction of the IP surface, occupied by two accretion columns. We derived $N_{\mathrm{e}}=$ $4.1-5.8 \times 10^{15} f_{-3}^{-1} \mathrm{~cm}^{-3}$ and $h_{\mathrm{s}}=0.21-0.28 f_{-3} R_{\mathrm{wd}}$, where $f_{-3}=10^{-3} f$ is the fraction of the surface of the IP, occupied by two accretion columns in units of $10^{-3}$. The derived parameters are similar to those obtained by De Martino et al. (2001) and Bonnet-Bidaud et al. (2001).
Acknowledgements. M.F. acknowledges the French Space Agency and CNRS for financial support. V.S. was partially supported by the Academy of Finland exchange grant. M.F. and V.S. are grateful to the NORDITA Nordic project on High Energy Astrophysics and the University of Oulu where part of this work was done.

\section{References}

Aizu, K. 1973, Prog. Theoret. Phys., 49, 1184

Arnaud, K. A. 1996, in Astronomical Data Analysis Software and Systems V, ed. G. H. Jacoby, \& J. Barnes (San Francisco: ASP), ASP Conf. Ser., 101, 17

Bonnet-Bidaud, J. M., Mouchet, M., de Martino, D., Matt, G., \& Motch, C. 2001, A\&A, 374, 1003

Courvoisier, T. J.-L., Walter, R., Beckmann, V., et al. 2003, A\&A, 411, L57

Cropper, M., Wu, K., Ramsay, G., \& Kocabiyik, A. 1999, MNRAS, 306, 684

Cropper, M. 1990, Space Sci. Rev., 54, 195

De Martino, D., Matt, G., Mukai, K., et al. 2001, A\&A, 377, 499

Downes, R., Webbink, R., F., \& Shara, M. M. 1997, PASP, 109, 345

Eckert, D., Walter, R., Kretschmar, P., et al. 2004, Astr. Tel., 352

Falanga, M., Kuiper, L., Poutanen, J., et al. 2005, A\&A, 444, 15

Fox, D. B., \& Kulkarni, S. R. 2004, Astr. Tel., 354

Frank, J., King, A., \& Raine, D. 2002, in Accretion Power in Astrophysics (Camb. Univ. Press)

Goldwurm, A., David, P., Foschini, L., et al. 2003, A\&A, 411, L223

Gros, A., Goldwurm, A., Cadolle-Bel, M., et al. 2003, A\&A, 411, L179

Haberl, F., \& Motch, C. 1995, A\&A, 297, L37

Kozhevnikov, V. P. 2001, A\&A, 366, 891

Lebrun, F., Leray, J.-P., Lavocate, Ph., et al. 2003, A\&A, 411, L141

Lund, N., Budtz-Jørgensen, C., Westergaard, N. J., et al. 2003, A\&A, 411, L231

Magdziarz, P., \& Zdziarski, A. A. 1995, MNRAS, 273, 837

Motch, C., Haberl, F., \& Guillout, P., et al. 1996, A\&A, 307, 459

Nauenberg, M. 1972, ApJ, 175, 417

Norton, A. J., Beardmore, A. P. Allan, A., \& Hellier, C. 1999, A\&A, 347, 203

Patterson, J. 1994, PASP, 106, 209

Revnivtsev, M., Lutovinov, A., Suleimanov, V., Sunyaev, R., \& Zheleznyakov, V. 2004, A\&A, 426, 253

Roques, J. P., Schanne, S., von Kienlin, A., et al. 2003, A\&A, 411, L91

Suleimanov, V., Revnivtsev, M., \& Ritter, H. 2005, A\&A, 435, 191

Ubertini, P., Lebrun, F., Di Cocco, G., et al. 2003, A\&A, 411, L131

Warner, B. 1995, Cataclysmic variable stars (Cambridge University Press)

Winkler, C., Courvoisier, T. J.-L., Di Cocco, G., et al. 2003, A\&A, 411, L1

Wu, K., Chanmugam, G., \& Shaviv, G. 1994, ApJ, 426, 664

Zombeck, M. V. 1990, Handbook of Astronomy \& Astrophysics (Cambridge: Cambridge Univ. Press) 